\title{
Perfil clínico de personalidad mediante el Inventario Clínico Multiaxial (MCMI-II) en pobla- ción ludópata del Instituto Nacional de Salud Mental Honorio Delgado - Hideyo Noguchi
}

\author{
Melissa Noemí PAREDES TORRES'1, Yanina SANDOVAL GUERRERO²
}

\begin{abstract}
RESUMEN
Objetivo: Determinar y describir el perfil clínico de la personalidad promedio, determinar el porcentaje de sujetos que presentan trastornos de personalidad, determinar la existencia de patrones clínicos y patologías severas de la personalidad e identificar la media de trastornos de la personalidad por sujeto. Metodología: El tipo de estudio fue cuantitativo y de nivel descriptivo. El diseño de la investigación fue no experimental, transversal y retrospectivo, realizado a 19 pacientes con diagnóstico de ludopatía, cuyas edades fluctuaron entre 18 y 60 años. Se empleó la adaptación española del Inventario Clínico Multiaxial de Millon (MCMI-II) de Kudó (1999). Se tomaron en cuenta solo los patrones clínicos y las patologías severas de la personalidad del MCMI-II. Además, se analizó su nivel de confiabilidad obteniendo un 0.77 para cada escala. Resultados: El 79\% de los sujetos estudiados presentaban al menos un trastorno de la personalidad. Además, las escalas que se presentaron con mayor frecuencia y que obtuvieron puntajes elevados significativos fueron: Agresivo- Sádico (52,6\%), Narcisista (42,1\%), Pasivo- Agresivo (42,1\%), Evitativo (36,8\%) y Antisocial (36,8\%). Por otro lado, se obtuvo como media 3,4 trastornos de personalidad por sujeto. Finalmente, se estableció el perfil clínico de la personalidad, tomando en cuenta las escalas con mayor prevalencia en la población de estudio. Conclusión: Un gran porcentaje de pacientes con diagnóstico de juego patológico presentaron comorbilidad con los trastornos de la personalidad.
\end{abstract}

Palabras Clave: Perfil clínico, patrones de personalidad, MCMI-II, patologías severas de personalidad, ludopatía, conducta de juego patológico.

\begin{abstract}
Objective: Identify and describe the clinical profile of the average personality, determine the percentage of subjects with personality disorders, determining the existence of severe clinical patterns and pathologies of the personality and the average iidentificar personality disorders per subject. Methods: The present study was quantitative and descriptive level. The research design was non-experimental, cross-sectional and retrospective. Consisted of 19 patients diagnosed with pathological gambling, whose ages ranged between 18 and 60. We used the Spanish adaptation of the Millon Clinical Multiaxial Inventory (MCMI-II) Kudo (1999). It took into account only the clinical disease patterns and severe pathologies of the personality of MCMI-II. Also analyzed for obtaining a confidence level 0.77 for each scale. Results: $79 \%$ of the subjects studied had at least one personality disorder. In addition, the scales were presented more frequently and that scored high were significant: Aggressive-Sadistic (52.6\%), narcissistic (42.1\%), Passive-Aggressive (42.1\%), Avoidant (36, 8\%) and Antisocial (36.8\%). On the other hand, we obtained an average of 3.4 personality disorders per subject. Finally, we established the clinical profile of the personality, taking into account the scale with the highest prevalence in the study population. Conclusions: A large percentage of patients with pathological gambling with comorbid personality disorders
\end{abstract}

Keywords: Clinical characteristics, patterns of personality, MCMI-II, severe pathology of personality, pathological gambling, pathological gambling behavior.

\footnotetext{
${ }^{1}$ Psicóloga de la ONG Asociación Pro Desarrollo Perú Vida "APRODE-PERU”

${ }^{2}$ Magíster en Psicología de la Salud, docente de la Facultad de Ciencias de la Salud, Universidad Peruana Unión.
} 


\section{INTRODUCCIÓN}

En los últimos años se ha producido un interés creciente por el estudio de la ludopatía como una nueva adicción a pesar de que éste es un fenómeno que ha estado presente desde tiempos antiguos en diversas culturas a través del mundo (Red Salud UC, 2010). Los estudios llevados a cabo han estimado que tanto en Europa como en Norteamérica, la prevalencia de este trastorno en la población es de un 2-3\%, y es dentro de este porcentaje que un $40 \%$ fluctúan entre los 18 y 30 años de edad (APA, 2005 y Chóliz, 2006). Sin embargo, en el Perú no encontramos datos estadísticos sobre la prevalencia de este trastorno. A pesar de esto es evidente que este no es un problema ajeno a nuestra población (Echeburúa, Fernández-Montalvo \& Báez, 2000).

Ahora bien, se hace inevitable no considerar esta situación como alarmante, debido a que las cifras estadísticas reportadas por investigadores de otros países en relación a este trastorno revelan que este fenómeno va en aumento y las edades de inicio cada año van disminuyendo, acarreando un grave problema de salud pública y mental que conlleva consecuencias perjudiciales y que afecta todos los ámbitos de la persona que lo padece, llevándolo a la pérdida de dinero, familia, trabajo y relaciones. Asimismo, es un factor de riesgo para el abuso de drogas o para padecer de ansiedad o depresión, que en ocasiones podría finalizar en un suicidio (Aranda, Díaz, García \& González, 1991; Báez, Borda, Salaverria, \& Echeburúa, 1996; Echeburúa, Fernández-Montalvo \& Báez, 2000, Muñoz, 2008 \& Bisso-Andrade, 2007).

Conociendo, pues, las graves repercusiones del Juego Patológico se considera necesario el estudio de este fenómeno con respecto al perfil clínico de la personalidad, ya que si bien es cierto existen estudios llevados a cabo en otros países, sus resultados son muy heterogéneos, no permitiéndonos establecer un perfil homogéneo de los Trastornos de la Personalidad en este tipo de pacientes $y$, por ende, no se puede brindar un tratamiento integral que busque la recuperación del paciente a través de diversos enfoques de tratamiento del juego patológico y que trate alguna otra patología asociada, como es el caso de los Trastornos de la Personalidad.

Por ello, el objetivo de esta investigación es conocer el perfil clínico de la personalidad en la población ludópata, a través de la medición con el Inventario Clínico Multiaxial de Millon (MCMI-II), el cual es un instrumento de autoinforme muy utilizado en la evaluación de los trastornos de personalidad y cuyo autor ha ejercido una influencia determinante en la clasificación misma de los trastornos de personalidad del DSM-IV-TR.

\section{MATERIAL Y MÉTODOS}

En esta investigación cuantitativa, descriptiva y retrospectiva se han identificado los perfiles clínicos de la personalidad en 19 pacientes con conducta de juego patológico, asistidos durante el período de febrero y diciembre del año 2010 en el I.N.S.M. Honorio Delgado-Hideyo Noguchi, a través del Inventario Clínico Multiaxial de Millon, elaborado por Kudó (1999), en la población con conducta de juego patológico.

El Inventario Clínico Multiaxial de Millon (MCMI-II), está conformado por 26 escalas diagnósticas, con 175 afirmaciones breves y auto descriptivas, las cuales se encuentran descritas en el Manual del MCMI-II; además, las descripciones más extensas acerca de la personalidad y su patología se encuentran en las obras de Millon. Igualmente, cabe resaltar que el MCMI-II se basa también en la descripción de los trastornos de la personalidad redactados en el DSM-III.

Los patrones clínicos de personalidad medidos fueron: Esquizoide, Evitativo, Dependiente, Histriónico, Narcisista, Antisocial, Agresivo-Sádico, Compulsivo, Pasivo-Agresivo y Autoderrotista. Las patologías severas de la personalidad medidas fueron: Esquizotípico, Borderline y Paranoide. Asimismo, el Coeficiente de alfa de Cronbach fue de 0.915 , lo cual indica que el instrumento tiene buena consistencia interna.

El procedimiento seguido ha sido el siguiente: (a) Completamiento de la Hoja de Respuestas del Inventario Clínico Multiaxial de Millon (MCMI-II), (b) Vaciado de los datos de la Hoja de Respuestas al Programa Computarizado de Kudó (1999), (c) Interpretación de Hoja de Resultados.

Asimismo, antes de la interpretación se evaluaron los puntajes de Validez de los resultados. Posterior a la evaluación de las escalas de Validez, se seleccionó las escalas con puntajes elevados para dar inicio a la etapa de interpretación. Para ello se analizaron los intervalos de los indicadores.

\begin{tabular}{|l|r|}
\hline Indicador Elevado: & $>=85$ \\
\hline Indicador Moderado: & $75-84$ \\
\hline Indicador Sugestivo: & $60-74$ \\
\hline
\end{tabular}

Se ha utilizado las frecuencias porcentuales, medias y desviaciones estándar. Asimismo, se ha aplicado la Prueba t de Student de diferencia de medias para grupos independientes, con un nivel de confianza al $95 \%$. 


\section{RESULTADOS}

Se ha observado que el $79 \%$ de los sujetos estudiados presentó al menos un trastorno de personalidad. Este resultado es casi similar al de Lesieur \& Blume (1990), que de los 141 sujetos en estudio el $71 \%$ de ellos presentaba al menos un TP. Sin embargo, los resultados difieren de los estudios de Blaszczynski y Steel (1998), los cuales analizaron a 82 sujetos encontrando que el 93\% presentaba un TP, porcentaje mayor al encontrado en nuestro estudio.
Además, Kruedelbach \& cl. (2006), estudiaron a 162 pacientes, de los cuales un $61,1 \%$ presentaba algún TP, mientras que en el estudio de Fernández-Montalvo $\&$ cl. (2001), un 32\% presentó al menos un trastorno de personalidad, porcentaje que disminuye aún más en el estudio de Specker, Carlson, Edmonson, Johnson, $\&$ Marcotte (1996), en el cual solo un 25\% presentaba un TP de un total de 40 sujetos estudiados. A continuación se presentan los resultados obtenidos en cuanto a porcentaje de sujetos según los indicadores del MCMI-II

\section{Tabla 1}

\section{Porcentaje de sujetos según escalas e indicadores del MCMI-II}

\begin{tabular}{lcccc}
\hline & \multicolumn{3}{c}{ INDICADORES } \\
\cline { 2 - 5 } ESCALAS & Nada & $\begin{array}{c}\text { Indicador } \\
\text { sugestivo }\end{array}$ & $\begin{array}{c}\text { Indicador } \\
\text { moderado }\end{array}$ & $\begin{array}{c}\text { Indicador } \\
\text { elevado }\end{array}$ \\
\cline { 2 - 5 } & $\%$ & $\%$ & $\%$ & $\%$ \\
Sinceridad & $3(15,8)$ & $10(52,6)$ & $2(10,5)$ & $4(21,1)$ \\
Deseabilidad social & $8(42.1)$ & $3(15.8)$ & $6(31.6)$ & $2(10.5)$ \\
Autodescalificación & $8(42.1)$ & $8(42.1)$ & $1(5.3)$ & $2(10.5)$ \\
PATRONES CLÍNICOS DE PERSNALIDAD & & & \\
Esquizoide & $3(15.8)$ & $9(47.4)$ & $2(10.5)$ & $5(26.3)$ \\
Evitativo & $3(15.8)$ & $6(31.6)$ & $3(15.8)$ & $7(36.8)^{*}$ \\
Dependiente & $7(36.8)$ & $4(21.1)$ & $4(21.1)$ & $4(21.1)^{*}$ \\
Histriónico & $5(26.3)$ & $9(47.4)$ & $3(15.8)$ & $2(10.5)^{*}$ \\
Narcisista & $3(15.8)$ & $5(26.39$ & $3(15.8)$ & $8(42.1)^{*}$ \\
Antisocial & $3(15.8)$ & $6(31.6)$ & $3(15.89$ & $7(36.8)^{*}$ \\
Agresivo-sádico & $3(15.8)$ & $6(31.6)$ & & $10(52.6)^{*}$ \\
Compulsivo & $4(21.1)$ & $9(47.4)$ & $3(15.8)$ & $3(15.8)$ \\
Pasivo-agresivo & $5(26.3)$ & $6(31.6)$ & & $8(42.1)^{*}$ \\
Autoderrotista & $1(5.3)$ & $11(57.9)$ & $4(21.1)$ & $3(15.8)$ \\
PATOLOGÍAS SEVERAS DE LA PERSONALIDAD & & $3(15.8)$ \\
Esquizotípico & $3(15.8)$ & $12(63.2)$ & $1(5.3)$ & $3(15.8)$ \\
Borderline & $2(10.5)$ & $13(68.4)$ & $1(5.3)$ & $2(10.5)$ \\
Paranoide & $3(15.8)$ & $12(63.2)$ & $2(10.5)$ & \\
\hline
\end{tabular}

En la tabla 1 se aprecia que 10 sujetos correspondientes al 52,6 \% del total de la población presentan TP AgresivoSádico, además, 8 sujetos $(42,1 \%)$ presentan TP Narcisista, de igual manera observamos que otros 8 sujetos puntúan alto en el TP Pasivo-Agresivo. Son 7 sujetos (36,8\%), que presentan TP Evitativo, el número se repite para el TP Antisocial. Un número menor de 5 sujetos (26,3\%) presentan TP Esquizoide, mientras que 4 sujetos $(21,1 \%)$ presentan TP Dependiente, seguidos por un número inferior de 3 sujetos $(15,8 \%)$ que presentan TP Compulsivo, el porcentaje de sujetos se repite para el TP Autodestructivo, Esquizotípico y Borderline; finalmente, son 2 sujetos $(10,5 \%)$ que presentan TP Histriónico, al igual que otros 2 sujetos $(10,5 \%)$ que se ubican en el TP Paranoide.

Los trastornos de la personalidad, con mayor prevalencia, son: Escala Agresivo-Sádico (52,6\%), Narcisista (42,1\%), Pasivo-Agresivo (42,1\%), Evitativo (36,8\%) y Antisocial (36,8\%). 
Tabla 2

Hallazgos comparativos en trabajos de investigación sobre los trastornos de la personalidad en sujetos con conducta de juego patológico

\begin{tabular}{|c|c|c|c|c|c|c|c|}
\hline $\begin{array}{l}\text { PATRONES Y } \\
\text { PATOLOGÍAS } \\
\text { SEVERAS DE LA } \\
\text { PERSONALIDAD }\end{array}$ & $\begin{array}{l}\text { Paredes } \\
\text { Torres } \\
(2011)\end{array}$ & $\begin{array}{l}\text { Vida } \\
\text { Libre } \\
(2009)\end{array}$ & $\begin{array}{l}\text { Montalvo } \\
\text { y Echeburúa } \\
(2006)\end{array}$ & $\begin{array}{c}\text { Fernández } \\
\text {-Montalvo } \\
\text { \& cl. } \\
(2001) \\
\end{array}$ & $\begin{array}{l}\text { Blaszczynski } \\
\text { \& Steel } \\
\text { (1998) }\end{array}$ & $\begin{array}{l}\text { Lesieur \& } \\
\text { Blume } \\
(1990)\end{array}$ & $\begin{array}{l}\text { Specker, } \\
\text { \& cl. } \\
(1996)\end{array}$ \\
\hline & 19 & & $\begin{array}{c}50 \\
\text { sujetos }\end{array}$ & $\begin{array}{c}50 \\
\text { sujetos }\end{array}$ & $\begin{array}{c}82 \\
\text { sujetos }\end{array}$ & $\begin{array}{c}105 \\
\text { sujetos }\end{array}$ & \\
\hline Equizoide & $26.3 \%(4)$ & $25 \%(3)$ & & & & & $2.5 \%$ \\
\hline Evitativo & $36.8 \%(3)$ & & & & & & $12.5 \%(1)$ \\
\hline Dependiente & $21.1 \%(5)$ & $28 \%(2)$ & & & $40.2 \%(4)$ & & \\
\hline Histriónico & $10.5 \%(7)$ & & & & $65.9 \%(2)$ & & \\
\hline Narcisista & $42.1 \%(2)$ & & $32 \%(1)$ & $8 \%(2)$ & $57 \%(3)$ & & $5 \%(2)$ \\
\hline Antisocial & $36.8 \%(3)$ & & $16 \%(2)$ & $8 \%(2)$ & $29.3 \%(5)$ & & \\
\hline Agresivo-sádico & $52.6 \%(1)$ & & & & & & \\
\hline Compulsivo & $15.8 \%(6)$ & $35 \%(1)$ & & & & $14 \%(2)$ & $5 \%(2)$ \\
\hline Pasivo-agresivo & $42.1 \%(2)$ & & $16 \%(2)$ & & & $14 \%(2)$ & \\
\hline Autoderrotista & $15.8 \%(6)$ & & & & & & \\
\hline Esquizotípico & $15.8 \%(6)$ & & & & & $28 \%(1)$ & \\
\hline Borderline & $15.8 \%(6)$ & & & $16 \%(1)$ & $69 \%(1)$ & $14 \%(2)$ & \\
\hline Paranoide & $10.5 \%(7)$ & $25 \%(3)$ & & $8 \%(2)$ & & & $2.5 \%(3)$ \\
\hline
\end{tabular}

En la tabla 2 podemos observar que los porcentajes de prevalencia de los patrones clínicos de personalidad son similares y en ocasiones iguales a los de otros estudios; por ejemplo: El patrón clínico Esquizoide 26.3\% (4) ubicado en 4to lugar de prevalencia, se asemeja a los resultados de Vida Libre, en el que obtiene un 25\% (3) y al estudio de Specker y cl. 2.5\% (3) en el cual el patrón clínico Esquizoide se ubica en el tercer lugar de prevalencia. Además, observamos que el patrón clínico Evitativo (36.8\%) (3) ubicado en el tercer lugar de prevalencia, difiere de ubicación en el estudio de Specker, \& cl. (1996), en el que el patrón clínico Evitativo (12.5\%) (1), se encuentra ubicado en el primer lugar de prevalencia. El patrón clínico Dependiente 21.1\% (5), situado en el quinto lugar de prevalencia, se asemeja con el resultado 40.2\% (4) de Blaszczynski \& Steel, el que se ubica en el cuarto lugar. El patrón clínico Histriónico (10.5\%) (7), ubicado en el séptimo lugar, se encuentra posicionado en el segundo lugar de prevalencia en el estudio de Blaszczynski \& Steel (1998). El patrón clínico Narcisista 42.1\% (2) prevalece en el segundo lugar al igual que en el estudio de Specker, \& cl. 5\% (2) y en el de Fernández -Montalvo \& cl. 8\% (2), y se asemeja a los resultados de Montalvo y Echeburúa 32\% (1), en el cual se ubica en el primer lugar de prevalencia y en el tercer lugar en el estudio de Blaszczynski \& Steel. 57\% (3). El patrón clínico Antisocial 36.8\% (3), ubicado en el tercer lugar, se asemeja con los hallazgos de Montalvo y Echeburúa 16\% (2) y con los resultados de la investigación de Fernández -Montalvo 8\% (2), en los que se ubica en el segundo lugar. El patrón clínico Agresivo- Sádico (52.6\% (1), con el mayor porcentaje de prevalencia en nuestro estudio, no ha sido referido en otros estudios. El patrón clínico Compulsivo (15.8\%) (6), ubicado en el sexto lugar en nuestro estudio, ha sido el patrón clínico con mayor prevalencia 35\% (1) en el estudio de Vida Libre (2009) y fue el segundo patrón con mayor prevalencia en los estudios de Lesieur \& Blume (1990) y en el de Specker, \& cl. (1996). Asimismo, encontramos que el patrón clínico Pasivo-agresivo $42.1 \%$ (2), situado en el segundo lugar, tiene igual prevalencia en los estudios de Montalvo y Echeburúa 16\% (2) y el de Lesieur \& Blume 14\% (2). El patrón clínico Autoderrotista (15.8\%) (6), ubicado en el sexto lugar, no ha sido referido en otros estudios. Por otro lado, al comparar los porcentajes de prevalencia de las patologías severas de la personalidad encontrados en nuestro estudio con los de otros estudios podemos observar que la patología severa de la personalidad Esquizotípico (15.8\%) (6), ubicado en el sexto lugar en nuestro estudio, se ubica en el primer lugar 
de prevalencia en el estudio de Lesieur \& Blume (1990). La patología severa Borderline 15.8\% (6), ubicado de igual manera en el sexto lugar de prevalencia en nuestro estudio, fue la patología severa con mayor prevalencia en el estudio de Fernández -Montalvo \& cl. (2001), con un 16\% y en el estudio de Blaszczynski \& Steel (1998) con un $69 \%$, además, de ubicarse en el segundo lugar de prevalencia con un 14\%, en el estudio de Lesieur \& Blume (1990). Finalmente, la patología severa Paranoide 10.5\% (7), prevalece en el tercer lugar en el estudio de Vida Libre (2009) con un $25 \%$ y en el estudio de Specker \& cl. (1996) con un $2.5 \%$, además se observó que fue la segunda patología con mayor porcentaje en el estudio de Fernández -Montalvo \& cl. (2001).

Tabla 3

Prueba t de Student de diferencia de medias de las diversas escalas de dos grupos independientes según el autor y lo reportado por Rojas y Espinoza (2002)

\begin{tabular}{|c|c|c|}
\hline ESCALAS & $\begin{array}{l}\text { COMPARACIÓN } \\
\text { REPORTADO } \\
\text { POR EL AUTOR }\end{array}$ & $\begin{array}{c}\text { REPORTADO POR } \\
\text { Rojas y Espinoza (2002) }\end{array}$ \\
\hline \multicolumn{3}{|l|}{ Escala de Validez } \\
\hline Sinceridad & 65 & 75,4 \\
\hline Deseabilidad social & 61 & 65,7 \\
\hline Autodescalificación & 55 & 61,0 \\
\hline \multicolumn{3}{|c|}{ Patrones clínicos de personalidad } \\
\hline Esquizoide & 73 & 62,8 \\
\hline Evitativo & $76^{*}$ & 70,1 \\
\hline Dependiente & 64 & 57,7 \\
\hline Histriónico & 63 & 78,2 \\
\hline Narcisista & $76^{*}$ & $89,1 *$ \\
\hline Antisocial & $75^{*}$ & $91,5^{*}$ \\
\hline Agresivo-sádico & $79 *$ & $93,3 *$ \\
\hline Compulsivo & 67 & 56,5 \\
\hline Pasivo-agresivo & 70 & $91,3 *$ \\
\hline Autoderrotista & $75^{*}$ & 73,1 \\
\hline \multicolumn{3}{|l|}{ Patología severa de personalidad } \\
\hline Esquizotípico & 69 & 65,9 \\
\hline Borderline & 69 & $80,7^{*}$ \\
\hline Paranoide & 68 & 73,1 \\
\hline MEDIA & 65 & 74,1 \\
\hline DESVIACIÓN ESTÁNDAR & 18.2 & 12,4 \\
\hline
\end{tabular}

En la Tabla 3, con respecto a la comparación de los hallazgos de Rojas y Espinoza (2002), se observa en un grupo de pacientes consumidores de drogas, encontramos lo siguiente: En la escala Evitativo se observa indicadores moderados (76), a diferencia del indicador sugestivo $(70,1)$ del estudio de Rojas y Espinoza, además, para la escala Narcisista, obtenemos indicadores moderados de (76), que difiere al indicador elevado $(89,1)$ del estudio comparativo; asimismo, obtenemos indicadores moderados para la escala Antisocial (75), que difieren del indicador elevado en esta escala $(91,5)$ del otro estudio; de igual manera, hallamos indicadores moderados en la escala Agresivo-sádico (79), e indicadores elevados para la misma escala $(93,3)$ en el estudio comparativo, finalmente, en la escala Autoderrotista obtenemos indicadores moderados (75), semejantes a los indicadores moderados para esta misma escala $(73,1)$ del estudio de Rojas y Espinoza. 
Figura 1

Perfil clínico según prevalencia de trastornos de la personalidad

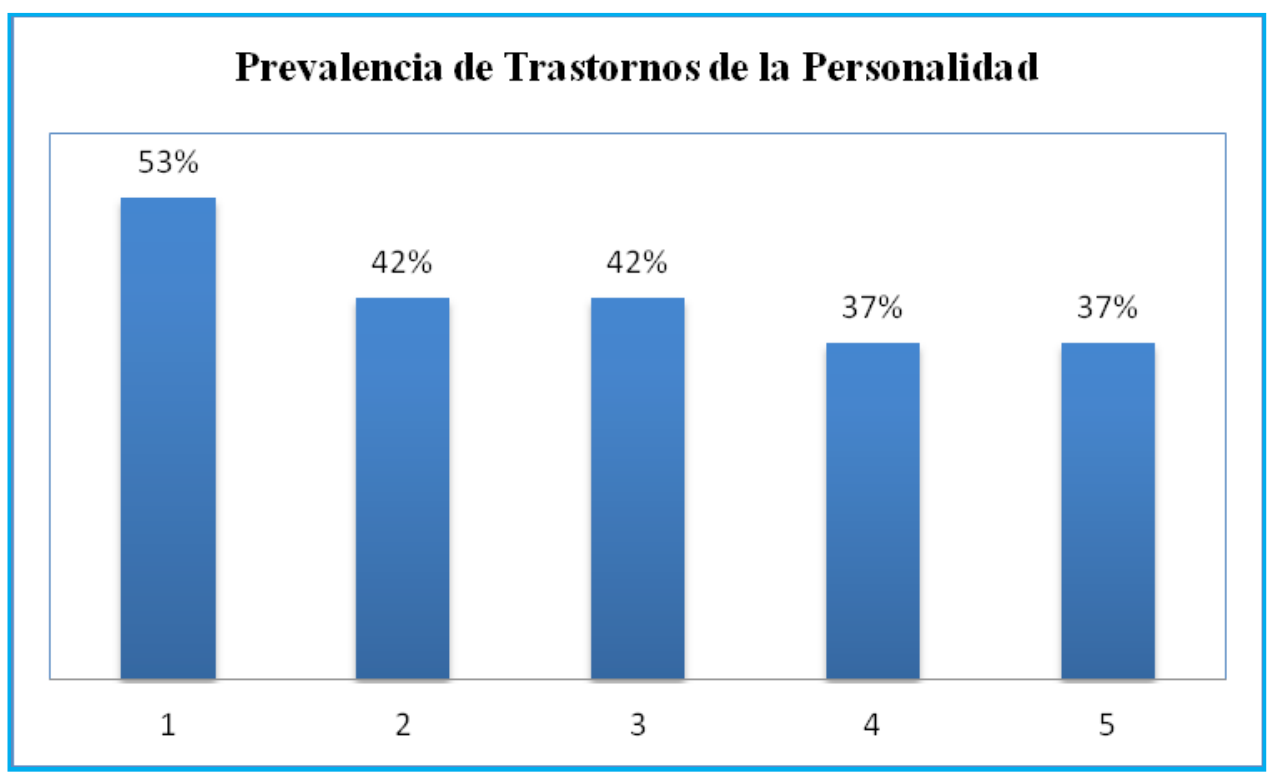

Leyenda:

1: Agresivo- sádico

2. Narcisista

3. Pasivo-Agresivo

4. Antisocial

5. Evitativo

De las prevalencias más significativas encontradas en nuestros resultados con respecto a los trastornos de la personalidad en el 79\% de la población. Podemos, establecer el perfil clínico, tomando en cuenta las puntuaciones elevadas en las siguientes escalas: Agresivo-sádico, Narcisista, Pasivo-agresivo, Evitativo y Antisocial. Por lo cual, el perfil clínico de personalidad hallado, describiría al sujeto ludópata como un sujeto arrogante, superficial, egocéntrico, intolerante, impulsivo y agresivo, con un temperamento exaltado y nervioso que estalla fácilmente en disputas y beligerancia física. Sus rasgos narcisistas develan su egoísmo y arrogancia, generados a través del consentimiento excesivo durante su crianza, llegando así a sobreestimar su propio valor, creyéndose superior a los demás y manteniendo un aire de autoconfianza arrogante. Ha aprendido a preocuparse a lo largo de su vida por suplir sus fantasías de belleza, amor y éxito. Por lo que busca constantemente la alabanza, el reconocimiento y admiración de los demás, ostentando sus logros y cualidades, a fin de ser reconocido, cuando no abusando y doblegando, con el objetivo de ser considerado superior a los otros. Sin embargo, ha sido también objeto de burlas y humillaciones en el pasado, generándole una marcada desconfianza e inseguridad ante las aspiraciones de los demás, actuando por medio de impulsos internos de agresión y venganza para contrarrestar las expectativas de dolor y depreciación de otros, realizando conductas que van desde resistirse a cumplir las expectativas de los demás, obstruyendo y minando las aspiraciones y satisfacciones de los otros, hasta conductas violentas que transgreden los derechos humanos, llegando a sentir placer al realizar este tipo de conductas, puesto que satisface sus impulsos de venganza y refuerza su percepción de ser superior a los demás. Finalmente, su carencia a gran escala de empatía, lo lleva a mostrarse ajeno e imperturbable ante las consecuencias de sus actos.

\section{DISCUSIÓN}

Con respecto al porcentaje de sujetos con trastornos de la personalidad, encontramos que el $79 \%$ de ellos presentaban, al menos, un trastorno de personalidad. El porcentaje hallado es muy similar al del estudio de Lesieur \& Blume (1990), en el que $71 \%$ de los sujetos presentaban al menos un TP. Sin embargo, nuestros resultados difieren del estudio de Blaszczynski y Steel (1998), los cuales encontraron que el 93\% de su población de estudio presentaba al menos un TP, así como también difieren, del estudio de Kruedelbach
\& cl. (2006), en el que se encontró que el $61,1 \%$ de su población, presentaba algún TP, mientras que en el estudio de Fernández-Montalvo \& cl. (2001), un 32\% de sujetos presentó al menos un trastorno de personalidad, porcentaje que disminuye aún más en el estudio de Specker, Carlson, Edmonson, Johnson, \& Marcotte (1996), en el cual solo un 25\% presentaba un TP.

Por otro lado, los porcentajes de prevalencia de los patrones clínicos de personalidad hallados son: Escala Esquizoide (26,3\%), Evitativo (36,8\%), Dependiente (21.1\%), Histriónico (10.5\%), Narcisista (42,1\%), An- 
tisocial (36,8\%), Agresivo-Sádico (52,6\%), Compulsivo $(15.8 \%)$, Pasivo-Agresivo $(42,1 \%)$, Autoderrotista (15.8\%), Esquizotípico (15.8\%), Borderline (15.8\%) y Paranoide (10.5\%). Sin embargo, al compararlos con los resultados de otros estudios acerca de este tema, encontramos lo siguiente: El patrón clínico Esquizoide $26.3 \%$ (4) ubicado en 4to lugar de prevalencia, se asemeja a los resultados de Vida Libre, en el que obtiene un $25 \%$ (3) y al estudio de Specker y cl. $2.5 \%$ (3) en el cual el patrón clínico Esquizoide se ubica en el tercer lugar de prevalencia. Además, observamos que el patrón clínico Evitativo (36.8\%) (3) ubicado en el tercer lugar de prevalencia, difiere de ubicación en el estudio de Specker, \& cl. (1996), en el que el patrón clínico Evitativo (12.5\%) (1), se encuentra ubicado en el primer lugar de prevalencia.

El patrón clínico Dependiente 21.1\% (5), ubicado en el quinto lugar de prevalencia, se asemeja con el resultado $40.2 \%$ (4) de Blaszczynski \& Steel, en el que se ubica en el cuarto lugar. El patrón clínico Histriónico (10.5\%) (7), ubicado en el séptimo lugar, se encuentra situado en el segundo lugar de prevalencia en el estudio de Blaszczynski \& Steel (1998). El patrón clínico Narcisista $42.1 \%$ (2) prevalence en el segundo lugar al igual que en el estudio de Specker, \& cl. 5\% (2) y en el de Fernández -Montalvo \& cl. 8\% (2), y se asemeja a los resultados de Montalvo y Echeburúa 32\% (1), en el cual se ubica en el primer lugar de prevalencia y en el tercer lugar en el estudio de Blaszczynski \& Steel. 57\% (3). El patrón clínico Antisocial 36.8\% (3), ubicado en el tercer lugar, se asemeja con los hallazgos de Montalvo y Echeburúa 16\% (2) y con los resultados de la investigación de Fernández -Montalvo 8\% (2), en los que se ubica en el segundo lugar. El patrón clínico Agresivo- Sádico (52.6\% (1), con el mayor porcentaje de prevalencia en nuestro estudio, no ha sido referido en otros estudios. El patrón clínico Compulsivo (15.8\%) (6), ubicado en el sexto lugar en nuestro estudio, ha sido el patrón clínico con mayor prevalencia $35 \%$ (1) en el estudio de Vida Libre (2009) y fue el segundo patrón con mayor prevalencia en los estudios de Lesieur \& Blume (1990) y en el de Specker, \& cl. (1996).

También, encontramos que el patrón clínico Pasivoagresivo $42.1 \%$ (2), situado en el segundo lugar, tiene igual prevalencia en los estudios de Montalvo y Echeburúa 16\% (2) y el de Lesieur \& Blume 14\% (2). El patrón clínico Autoderrotista (15.8\%) (6), ubi- cado en el sexto lugar, no ha sido referido en otros estudios.

Asimismo, los porcentajes de prevalencia de las patologías severas de la personalidad son: Esquizotípico (15.8\%) (6), ubicado en el primer lugar de prevalencia en el estudio de Lesieur \& Blume (1990). La patología severa Borderline 15.8\% (6), fue la patología severa con mayor prevalencia en el estudio de Fernández -Montalvo \& cl. (2001), con un 16\% y en el estudio de Blaszczynski \& Steel (1998) con un 69\%, además, de situarse en el segundo lugar de prevalencia con un 14\%, en el estudio de Lesieur \& Blume (1990). Finalmente, la patología severa Paranoide $10.5 \%$ (7), prevalece en el tercer lugar en el estudio de Vida Libre (2009) con un $25 \%$ y en el estudio de Specker \& cl. (1996) con un $2.5 \%$, además se observó que fue la segunda patología con mayor porcentaje en el estudio de Fernández-Montalvo \& cl. (2001).

Finalmente, en nuestros resultados encontramos como media 3,4 trastornos de personalidad por sujeto, resultado similar al del estudio de Vida Libre, en el cual también, se empleó el Inventario Clínico Multiaxial de Millon (MCMI-II), y en el que encontraron como media 2,8 trastornos de personalidad por sujeto (Montesinos, 2009).

\section{CONCLUSIONES}

- De la población de estudio, el $79 \%$ de los sujetos presentó trastornos de la personalidad.

- Los patrones clínicos que se han observado en la población de estudio en orden decreciente fueron: Agresivo-Sádico (52,6\%), Narcisista (42,1\%), Pasivo-Agresivo (42,1\%), Evitativo (36,8\%), Antisocial (36,8\%), Esquizoide (26,3\%), Dependiente $(21,1 \%)$, Compulsivo, $(15,8 \%)$, Autodestructivo $(15,8 \%)$ e Histriónico (10,5\%).

- Las patologías severas que se observaron en la población de estudio en orden decreciente fueron: Esquizotípico (15,8\%), Borderline $(15,8 \%)$ y Paranoide $(10,5 \%)$.

- Obtuvimos como media 3,4 trastornos de personalidad por sujeto.

- De las prevalencias más significativas encontradas en nuestros resultados con respecto a los trastornos de la personalidad se estableció el perfil clínico, tomando en cuenta las puntuaciones elevadas en las siguientes escalas: Agresivo-Sádico, Narcisista, Pasivo-Agresivo, Evitativo y Antisocial. 


\section{REFERENCIAS BIBLIOGRÁFICAS}

1. APA, A. P. (2005). DSM-IV-TR: Manual diagnóstico de los trastornos mentales. Barcelona, España.: Masson.

2. Aranda, J., Díaz, C., García, J., \& González, J. (1991). Evaluación Cognitivo-Conductual del Juego Patológico. Revista Clínica y Salud. Vol. 2. , 2 .

3. Báez, C., Borda, M., Salaberría, K., \& Echeburúa, E. (1996). El juego patológico: un análisis bibliométrico. Revista Clínica y Salud. Vol. 7. , 123-135.

4. Blaszczynski, A., \& Steel, Z. (1998). A pathways model of problem and pathological gambling. Addicition , 51-71.

5. Blaszczynski, A., Wilson, A., \& McConaghy, N. (1986.). Sensation seeking and pathological gambling. British Journal of Addictions. , 113117.

6. Brown, R., \& Robertson, S. (1993). Home computer and video game addictions in relation to adolescent gambling: Conceptual and developmental aspects. Gambling behavior and problem gambling.

7. Chóliz, M. (2008 de Agosto de 2006). Adicción al juego de azar. Recuperado el 12 de Marzo de 2010, de www. uv.es/choliz.

8. Echeburúa, E., \& Fernández-Montalvo, J. (2005). Psychological treatment of slot-machine pathological gambling: New perspectives. Journal of Gambling Studies. Vol. 21. , 21-26.

9. Echeburúa, E., Fernández-Montalvo, J., \& Báez, C. (2000). El juego patológico en España: aportaciones de un equipo de investigación en la última década (1990-2000). Revista de Psicología General y Aplicada. Vol. 53. , 641-660.

10. Estévez, A., \& Calvete, E. (2007). Esquemas cognitivos en personas con conducta de juego patológico y su relación con experiencias de crianza. Revista Clínica y Salud Vol.18 n.1. Madrid, España. , 2-4.
11. Fals-Stewart,W.(1992).Personality characteristics of substance abusers: An MCMI cluster typology of recreational drug users treated in a therapeutic community and its relationship to length of stay and outcome. Journal of Personality Assessment. 515-527.

12. Fenández-Montalvo, J., \& Echeburúa, E. (2004). Pathological gambling and personality disorders: an exploratory study with the IPDE. Journal of Personality Disorders. 500-505.

13. Fernández Montalvo, J., \& Echeburúa, E. (2006). Juego patológico y trastornos de personalidad: un estudio piloto con el MCMI-II. Psicothema.Vol. $18, n^{\circ} 3 ., 453-457$.

14. Fernández, M. J., \& Echeburúa, E. (2001). Trastornos de la Personalidad y Juego Patológico. Psicología Conductual. Vol.9, 527-539.

15. Fernández-Montalvo, J., \& Echeburúa, E. (2001). Trastornos de la personalidad y juego patológico: una revisión crítica. Psicología Conductual, 9. , 527-539.

16. Flynn, P. M., Luckey, J. W., Brown, B. S., Hoffman, J. A., \& al., e. (1995.). Relationship between drug preference and indicators of psychiatric impairment. American Journal of Drug \& Alcohol. , 153-166.

17. Ibánez, A., \& Saíz, J. (2000.). La Ludopatía: una nueva enfermedad. Barcelona, España.: Masson.

18. Ibañez Cuadrado, A., \& Sáiz Ruiz, J. (2001). La ludopatía una "nueva” enfermedad. Barcelona, España.: Masson.

19. Jacobs, D. (1986). A general theory of addictions: A new theoretical model. Journal of Gambling Behavior. Vol. 2 , 15-31.

20. Kruedelbach, N., Walker, H., Chapman, H., Haro, G., Mateu, C., \& Leal, C. (2006). Comoribilidad de trastornos con pérdidas del control de impulsos: ludopatìa, adicciones y trastornos de la personalidad. Actas Españolas de Psiquiatrìa. , 2. 
21. Lesieur, H., \& Blume, S. B. (1990). Characteristics of pathological gamblers identified patiens on a psychiatric admission service. Hospital and Community Psychiatry, 41.

22. Litman, L., \& Cernovsky, Z. (1993). An MCMII taxonomy of substance abusers. Research Communications in Psychology, Psychiatric \& Behavior. , 67-72.

23. Livesley, W. J. (1998). Suggestions for a framework for an empirically based classification of personality disorder. Canadian Journal of Psychiatric. Vo. 43 , 137-147.

24. López, J., \& Valdés, M. (2002). Manual de Diagnóstico y Estadistico de los Trastornos Mentales. Barcelona, España: Masson.

25. McCormick, R., \& Ramirez, L. (1988). Pathological gambling. New York. United States.: Brunner \& Mazel.

26. Millon, T., Davis, R., Millon, C., Escovar, L., \& Meagher, S. (2001). Trastornos de la Personalidad en la Vida Moderna. Barcelona, España.: Masson.

27. Millon, T., George, S., \& Everly, J. (1994). La Personalidad y sus Trastornos. Barcelona, España.: Ediciones Martínez Roca.

28. Montesinos Espí, R. (3 de abril de 2009). Vida Libre. AsociaciónAlicantina deAfectadospor la Ludopatía y otras Adicciones. Recuperado el 3 de diciembre de
2010, de Conselleria de Sanitat. Dirección General de Drogodependencias.: http://www.vidalibrealicante.org/4MEMOCaratpsicopatestudio.pdf.

29. Morán, E. (1970). Clinical and social aspects of risk-taking. United States.: Proc Royal Society of Medicine.

30. Muñoz-Molina, Y. (2008). Meta-análisis sobre juego patológico. Revista de Salud Pública. , 150159.

31. Oldhman, J. M. (2007). Tratado de los Trastornos de la Personalidad.The American Psychiatric Publishing. Madrid, España.: Elsevier España.

32. OMS. (2004). Clasificación de los Trastornos Mentales y del Comportamiento. Madrid, España.: Editorial Médica Panamericana.

33. Rojas, M., \& Espinoza, L. (20 de $\mathrm{m}$ arzo de 2002). Recuperado el 3 de diciembre de 2011, de Revista Científica Psicoactiva: http://www.cedro. org.pe/ebooks/Psicoactica_20.pdf

34. Rosenthal, R. (1987). The psychodynamics of pathological gambling: A review of the litereture. . Springfield: Ediciones Springfield.

35. Sánchez, E. (2003.). Juego Patológico: Un Trastorno Emergente. Unidad de Adictivas de Catarroja. Valencia, 30-79. 